\title{
Minimally invasive video assisted surgical closure of secundum atrial septal defect
}

\author{
Harold M. Burkhart ${ }^{1}$, Rakesh M. Suri ${ }^{2}$ \\ ${ }^{1}$ Division of Cardiovascular and Thoracic Surgery, University of Oklahoma Health Sciences Center, Oklahoma City, OK, USA; ${ }^{2}$ Department of \\ Cardiothoracic and Vascular Surgery, Cleveland Clinic Foundation, Cleveland, OH, USA \\ Correspondence to: Harold M. Burkhart, MD. Professor and Chief, Division of Cardiovascular and Thoracic Surgery, University of Oklahoma Health \\ Sciences Center, P.O. Box 26901, WP-2230, Oklahoma City, OK 73105, USA. Email: Harold-burkhart@ouhsc.edu.
}

Submitted Nov 17, 2016. Accepted for publication Dec 30, 2016.

doi: $10.21037 / \mathrm{acs} .2017 .01 .05$

View this article at: http://dx.doi.org/10.21037/acs.2017.01.05

\section{Clinical vignette}

A 53-year-old male presented with an eight-month history of shortness of breath and palpitations. Additionally, he had a recent history of paroxysmal atrial fibrillation. An echocardiogram demonstrated a large secundum atrial septal defect (ASD), a severely dilated right atrium and ventricle, and evidence of depressed right ventricular function (Figure 1). He was referred for ASD repair, biatrial maze and left atrial appendage ligation.

\section{Surgical techniques}

\section{Preparation}

The patient underwent single lung general endotracheal anesthesia utilizing either a double lumen tube or endobronchial blocker. A left radial arterial line and central venous catheter were placed for monitoring purposes. In addition, a right internal jugular venous sheath was placed just cephalad to the clavicle and prepped into the sterile surgical field. This sheath provides access for percutaneous jugular venous cannulation for cardiopulmonary bypass. Positioning for video-assisted thoracoscopic surgery (VATS) ASD repair involves a bolster placed medially to the right scapula to position the chest at a 30-degree angle to the operating table. The right arm is tucked posterior to the axillary line to allow for transthoracic aortic cross clamp placement. Care should be taken to ensure the head and neck are supported to avoid brachial plexus strain.

\section{Exposition}

The incisions and port placement are similar to the robotic mitral valve repair incisions (1). The robotic surgeon and another fully qualified bedside cardiovascular surgeon work together to ensure a safe and efficient repair. The right femoral vessels are exposed through a $1.5-\mathrm{cm}$ incision and purse strings are placed in the anterior common femoral artery and vein. The right lung is deflated and a $2.5-\mathrm{cm}$ incision is made in the right inframammary crease. The pleural space is entered over the fifth rib. A peripheral catheter is placed medial to the incision for the instillation of $\mathrm{CO}_{2}(10 \mathrm{mmHg})$. Next, the pericardium is opened $4 \mathrm{~cm}$ anterior to the phrenic nerve, revealing the aorta and right atrium. Pericardial sutures are placed on the inferior edge and pulled through a needle hole laterally at the axillary line.

Using the Seldinger technique and echocardiographic guidance, a 22- or 25-F (Edwards Lifesciences CardioVations, Irvine, California) venous cannula is inserted into the femoral vein and advanced to $1 \mathrm{~cm}$ below the right atrial inferior vena cava junction (Figures 2-4). A 16- or 18-F cannula is also inserted percutaneously via the previously described jugular venous sheath and advanced into the superior vena cava. Finally, an appropriately sized femoral arterial cannula is placed via the common femoral artery with echocardiographic guidance to confirm that the wire remains in true lumen of the aorta. Cardiopulmonary bypass is instituted over 2 minutes after adequate anticoagulation is confirmed. A long tack vent cannula (Medtronic, Minneapolis, Minnesota) is placed through the inframammary incision into an aortic purse string. Taking care to avoid injury to the right pulmonary artery, the aorta is cross-clamped. One liter of cold blood cardioplegia is given for cardiac arrest and smaller doses are repeated every 20 minutes while the cross clamp is in place. 


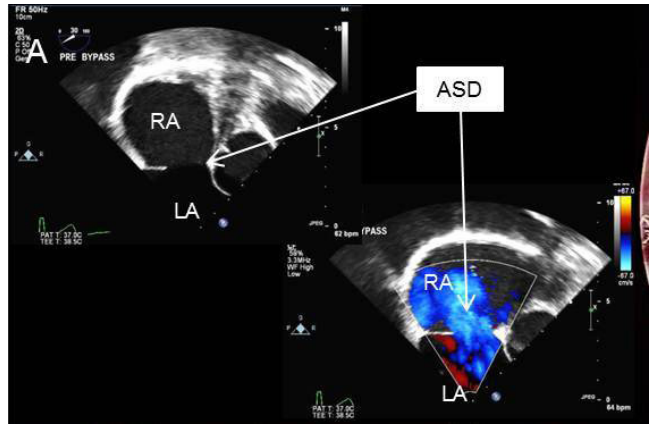

Figure 1 Transesophageal echocardiogram showing large secundum atrial septal defect. ASD, atrial septal defect; LA, left atrium; RA, right atrium.

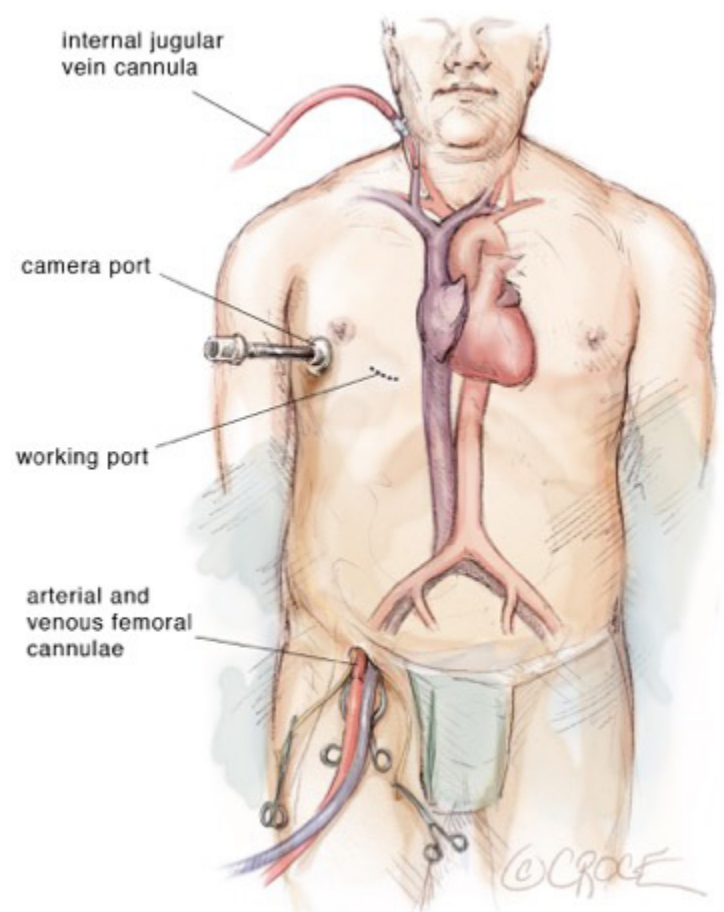

Figure 2 Diagram showing surgical set-up and location of cardiopulmonary bypass lines.

\section{Operation}

The superior vena cava is controlled with a bulldog vascular clamp and the inferior vena cava is occluded with a tourniquet. The right atrium is opened in an oblique fashion towards the atrial appendage. The atrial septum is inspected for the presence of more than one septal defect as well as septal aneurysm (Figure 5). Anomalous pulmonary venous drainage is ruled out. In a smaller defect or patent foramen

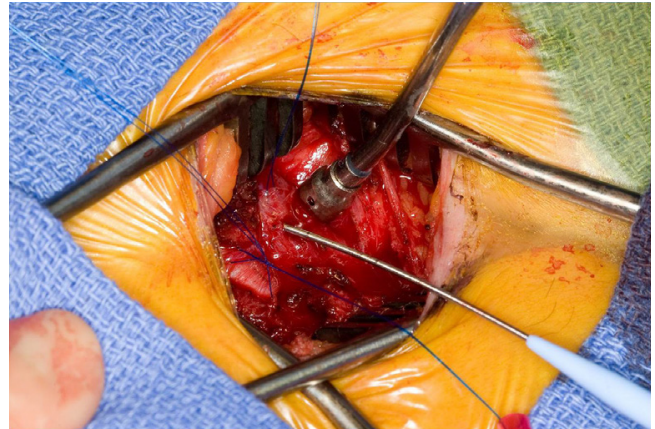

Figure 3 The femoral vein and artery have been exposed and purse strings placed. A guide wire has been inserted into the femoral vein.

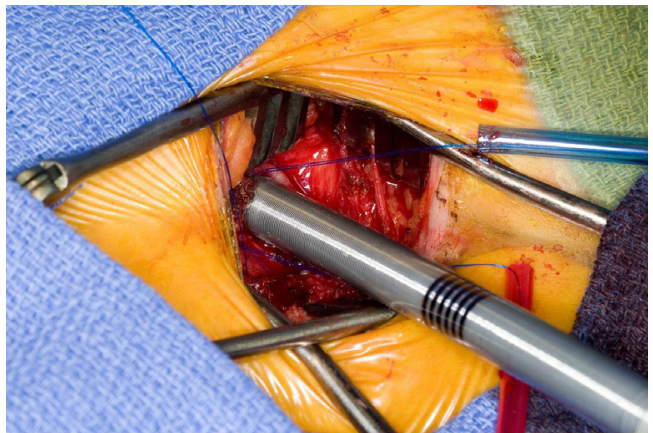

Figure 4 Utilizing the Seldinger technique with echocardiogram guidance, the femoral venous cannula is placed.

ovale (PFO), the septum can typically be closed primarily with two layers of non-absorbable polypropylene suture (3-0 Prolene, Ethicon Inc., Cornelia. Georgia, USA). In larger defects, a patch (e.g., autologous pericardium, bovine pericardium) is utilized with running sutures. If the septum is aneurysmal or has multiple defects, it should be excised and a patch utilized for reconstruction. Prior to tying down the repair suture, the left atrium is allowed to fill with blood and allow for air evacuation. Once the septum is intact, the aortic cross clamp is removed with the aortic tact vent kept in place for de-airing. The right atrium is repaired with two layers of running non-absorbable polypropylene sutures (3-0 Prolene, Ethicon Inc., Cornelia, Georgia, USA).

\section{Completion}

The integrity of the repair and adequacy of deairing is confirmed by transesophageal echocardiography. A bubble study is typically performed to rule out any residual shunt (Figure 6). Once satisfied with the result, cardiopulmonary 


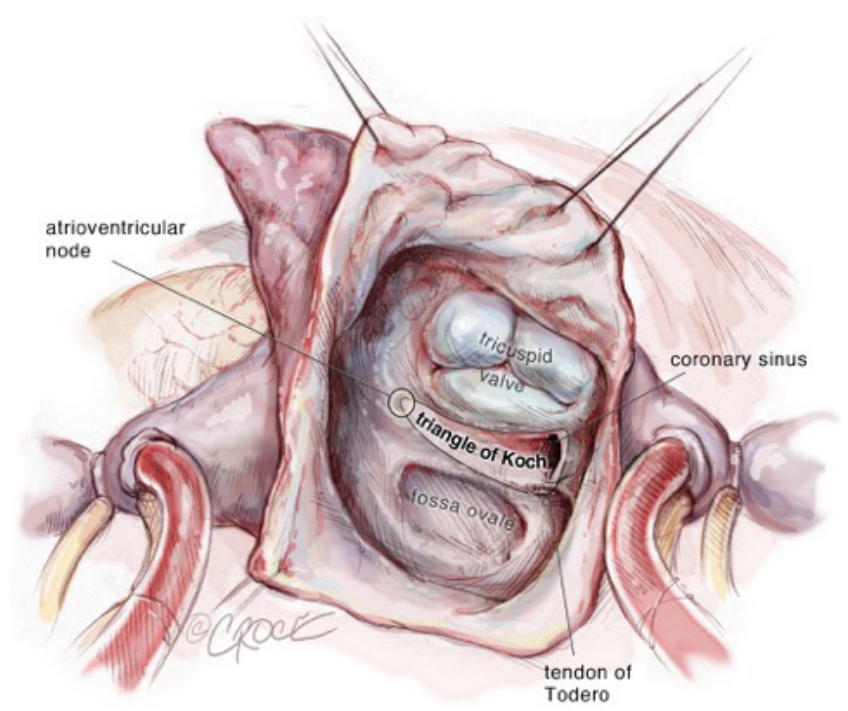

Figure 5 The anatomy of the atrial septum is demonstrated. Note the close proximity of the atrioventricular node.

bypass is temporarily reinstituted for removal of the cardioplegia tack vent and tying of the ascending aortic stitch. Decannulation and reversal of heparin are performed in the usual manner. The pericardium is loosely reapproximated and the chest and femoral wounds are closed in layers.

\section{Comments}

\section{Clinical results}

We have previously reported our early experience with 51 patients who underwent VATS ASD/PFO closure, with or without robotic assistance (2). Utilizing 2:1 frequencymatching, they were compared to 102 patients who underwent a traditional median sternotomy for defect closure. Median age was 43 years (3-71 years) and $67 \%$ were female. There were no conversions to sternotomy in the VATS group. Early mortality was $0 \%$ in both groups. Cross-clamp time $(14.5 \pm 7.6$ vs. $26.3 \pm 13.2 \mathrm{~min}, \mathrm{P}<0.001)$ and bypass time $(31.7 \pm 13.8$ vs. $60.9 \pm 20.9 \mathrm{~min}, \mathrm{P}<0.001)$ were longer in the VATS group. However, VATS patients had a shorter postoperative ventilation time $(7.5 \pm 6.4 \mathrm{vs}$. $4.4 \pm 2.8 \mathrm{~h}, \mathrm{P}=0.03$ ) with $62.7 \%$ extubated in the operating room along with shorter intensive care unit stay $(26.7 \pm 10.8$ vs. $19.1 \pm 9.9 \mathrm{~h}, \mathrm{P}<0.001)$ and hospital stay $(5.2 \pm 1.9$ vs. $3.5 \pm 0.9$ days, $\mathrm{P}<0.001$ ). At early follow-up (maximum 4.2 years), there was no difference in need for reintervention.

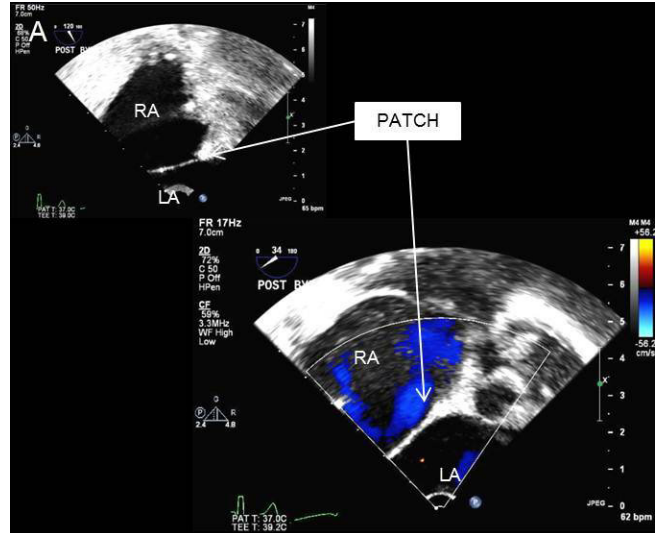

Figure 6 Transesophageal echocardiogram demonstrates an intact repaired atrial septum. LA, left atrium; RA, right atrium.

\section{Advantages}

The advent of VATS, with or without robotic assistance, avoids the disadvantages of conventional full sternotomy. The VATS approach has been reported to be safe and effective, with no mortality and very low morbidity (3). It requires expertise in both robotic and port-access techniques and as well as perfusion strategy. Improvement in postoperative recovery, expeditious return to work as well as improved cosmesis are evidence that a VATS or robotic approach is cost effective (4).

\section{Caveats}

We have expanded our use of VATS with robotic assistance to more complex congenital patients that benefit from the improved visualization and improved technical capabilities. This includes performing arrhythmia surgery as well as tricuspid valve repair along with the septal defect. Other cases include sinus venosus atrial septal defects as well as partial atrioventricular canal defects (5).

\section{Acknowledgements}

None.

\section{Footnote}

Conflicts of Interest: The authors have no conflicts of interest to declare. 


\section{References}

1. Suri RM, Burkhart HM. Optimizing outcomes of robotic mitral valve repair for all prolapse anatomy: the SuriBurkhart technique. Ann Cardiothorac Surg 2013;2:841-5.

2. Sabate Rotes A, Burkhart HM, Suri RM, et al. Minimally invasive video-assisted surgical closure of atrial septal defects: a safe approach. World J Pediatr Congenit Heart Surg 2014;5:527-33.

3. Bonaros N, Schachner T, Oehlinger A, et al. Robotically

Cite this article as: Burkhart HM, Suri RM. Minimally invasive video assisted surgical closure of secundum atrial septal defect. Ann Cardiothorac Surg 2017;6(1):60-63. doi: 10.21037/ acs.2017.01.05 assisted totally endoscopic atrial septal defect repair: insights from operative times, learning curves, and clinical outcome. Ann Thorac Surg 2006;82:687-93.

4. Suri RM, Thompson JE, Burkhart HM, et al. Improving affordability through innovation in the surgical treatment of mitral valve disease. Mayo Clin Proc 2013;88:1075-84.

5. Raju V, Burkhart HM, Cetta F Jr, et al. Successful robotassisted repair of congenital mitral valve regurgitation. Ann Thorac Surg 2014;98:1085-7. 